might be a consequence of aberrant nucleic acid editing. The 129-Ter mouse strain models pediatric TGCT in humans. Although germ cell tumors present clinically in infants and young adults, it is noted that the susceptibility of PGCs to tumorigenesis is dependent on both genetic and environmental influences during embryogenesis.

The authors conclude that the discovery of the Ter mutation in the Dnd1 gene will have important implications for understanding the genetic control of TGCT and PGC biology.

Original article Youngren KK et al. (2005) The Ter mutation in the dead end gene causes germ cell loss and testicular germ cell tumors. Nature 435: 360-364

\section{Prenatal phthalate exposure decreases anogenital distance in human males}

Phthalates are diesters of phthalic acid, and are widely used in personal care products, plastics and paints. Consistent toxicologic evidence indicates an association between phthalates and reproductive effects: phthalates have been shown to disrupt reproductive tract development in male rodents in an antiandrogenic manner, and prenatal exposure to high doses has resulted in significant reductions in the anogenital distance (AGD) in Sprague-Dawley rats. Despite the growing body of literature on extensive human exposure to phthalates and their reproductive toxicity, few studies have examined the effects of phthalates on human reproductive development.

In a multicenter pregnancy cohort study, the Study for Future Families II (SFFII), Swan et al. examined the effects of prenatal phthalate exposure on AGD and other genital measurements. Prenatal urine samples were assayed for nine phthalate metabolites in 85 mothers, and 134 boys aged 2-30 months received a breast and genital examination. AGD was significantly correlated with penile volume and the proportion of boys with incomplete testicular descent. There were no frank malformations or disease in any of the boys.

Urinary concentrations of four phthalate metabolites, mono-ethyl phthalate, mono-nbutyl phthalate, mono-benzyl phthalate and mono-isobutyl phthalate, were inversely related to anogenital index (AGI: the AGD divided by weight at examination), and the age-adjusted AGI decreased significantly with increasing combined exposure to these four metabolites. In boys whose mothers had elevated prenatal phthalate exposure, AGI was shortened and testicular descent was impaired. According to the National Health and Nutrition Examination Survey 1999-2000, the median concentrations of phthalate metabolites that are associated with short AGI and testicular impairment in this study are lower than those measured in $25 \%$ of the US female population. These findings support the hypothesis that prenatal phthalate exposure at environmental levels can adversely affect reproductive development in human males.

Original article Swan SH et al. (2005) Decrease in anogenital distance among male infants with prenatal phthalate exposure. Environ Health Perspect [doi:10.1289/ehp.8100]

\section{Predictive value of procalcitonin for vesicoureteral reflux}

Children presenting with a first febrile urinary tract infection are currently recommended to undergo systemic voiding cystourethrography, a painful and irradiating procedure used to diagnose vesicoureteral reflux (VUR) that in 60$80 \%$ of cases reveals no evidence of the condition. Leroy et al. investigated the relationship between procalcitonin (PCT) blood concentrations and VUR in children with a first febrile urinary tract infection, to determine whether PCT was able to predict the presence of VUR.

The authors carried out a retrospective study of 136 children aged between 1 month and 4 years of age who had suffered a first febrile urinary tract infection, $25 \%$ of whom were found to have VUR following systemic voiding cystourethrography. Serum PCT concentrations had been prospectively measured at admission. Univariate and multivariate statistical analysis was carried out to examine the nature of any relationship between grades of reflux and serum concentrations of PCT, and it was found that the median PCT concentration was significantly higher in children with VUR than in those without. The authors found that the relationship between these two variables strengthened in an almost linear fashion as the grade of VUR increased, so that a high PCT level predicted all-grade VUR with $>80 \%$ 